\title{
Shear Viscosity and Phase Diagram from Polyakov-Nambu-Jona-Lasinio model
}

\author{
Sanjay K. Ghosh $⿴$ Sibaji Raha \\ Center for Astroparticle Physics \& Space Science, \\ Block-EN, Sector-V, Salt Lake, Kolkata-700091, INDIA \\ E. \\ Department of Physics, Bose Institute, \\ 93/1, A. P. C Road, Kolkata - 700009, INDIA
}

\begin{abstract}
We discuss a detailed study of the variation of shear viscosity, $\eta$, with temperature and baryon chemical potential within the framework of Polyakov-Nambu-Jona-Lasinio model. $\eta$ is found to depend strongly on the spectral width of the quasi-particles present in the model. The variation of $\eta$ across the phase diagram has distinctive features for different kinds of transitions. These variations have been used to study the possible location of the Critical End Point (CEP), and cross-checked with similar studies of variation of specific heat. Finally using a parameterization of freeze-out surface in heavy-ion collision experiments, the variation of shear viscosity to entropy ratio has also been discussed as a function of the center of mass energy of collisions.
\end{abstract}

PACS numbers: 12.38.Aw, 12.38.Mh, 12.39.-x

\section{INTRODUCTION}

The relativistic heavy ion collision experiments provide us with the unique opportunity to understand the physics of strongly interacting matter expected to be present in the universe after a few microseconds of the big bang, and possibly present in the interior of neutron stars. In the experiments, two heavy ions colliding at relativistic energies are expected to form a fireball consisting of deconfined quarks and gluons, popularly known as the Quark-gluon plasma (QGP). The search for QGP is continuing for almost last thirty years using several generations of higher energy accelerators such as BEVALAC, AGS, SPS, RHIC and LHC while covering a large energy range of few AGeV to few ATeV. Various observables, such as, $J / \Psi$ suppression [1] and strangeness enhancement [2] had been proposed as signatures of such state of matter. All such proposed signatures are based on medium's properties which differed substantially in hadronic and quark phases. Charmonium suppression, for example, was based on the properties of deconfinement and plasma screening [1], whereas, strangeness enhancement was based on the chiral symmetry restoration, which may be fully realized in QGP but only partially in a hadron gas [2, 3].

If the main interest lies in the identification of a new form of bulk matter then it is essential to choose observables corresponding to unique collective properties of this matter. For example radial, azimuthal and longitudinal flow are some of the relevant observables in heavy ion collisions [4]. In general such observables may be obtained from azimuthal Fourier components [5], $v_{n}\left(y, p_{T}, N_{p}, h\right)$ of triple differential inclusive distribution of hadrons which are selected based on their impact parameter range.

The observation of elliptic flow in non-central heavy-ion collisions at RHIC, may be considered as the most important evidence for hydrodynamical behavior of QGP. Elliptic flow occurs when the plasma collectively responds to pressure gradients in the initial state. Hydrodynamic evolution converts the initial pressure gradients to velocity gradients in the final state. In a heavy-ion collision one cannot control the deformation of the initial state. Instead, the deformation of the plasma is determined by the shape of the overlapping region of the colliding nuclei. This shape is governed by the impact parameter $b$. The impact parameter can be measured on an event-by-event basis using the azimuthal dependence of the spectra of produced particles. Once the impact parameter direction is known, the particle distribution can be expanded in Fourier components of the azimuthal angle $\phi$. The Fourier coefficients $\left(v_{2}\right.$, $v_{4}$ etc.) carry information about the deformation of the final state. For example, a positive $v_{2}$ implies the preferential emission of particles in the short direction, i.e. the presence of elliptic flow. Since shear viscosity $\eta$ is expected to oppose the elliptic flow (and reduce $v_{2}$ ), it is necessary to incorporate $\eta$ in the analysis. Moreover a dimensionless

\footnotetext{
*Electronic address: sanjay@jcbose.ac.in

†Electronic address: sibaji@jcbose.ac.in

$\ddagger$ Electronic address: rajarshi@jcbose.ac.in

$\S$ Electronic address: saha.k.09@gmail.com

ๆ Electronic address: sudipa.09@gmail.com
} 
quantity $\eta / s$, where $s$ is the entropy density, tells us about the actual behavior of the fluid. The ratio $\eta / s$ is akin to the inverse of Reynolds number.

In general $\eta$ for a system of quasi-particles is expected to vary as $\sim \epsilon t_{m f t}$, where $\epsilon$ is the energy density and $t_{m f t}$ is the average mean free time. The entropy density $s \sim k_{B} n$, where $k_{B}$ is the Boltzmann constant and $n$ the number density. Since $\frac{\epsilon}{n}$ is the average energy per particle, considering uncertainty principle one would get a lower bound on the product of $\epsilon / n$ and $t_{m f t}$. In other words one would get $\eta / s \geq \frac{\hbar}{k_{B}}$. For the strong coupling limit of superconformal-QCD, Policastro et al. [6] found $\eta / s=\frac{\hbar}{4 \pi k_{B}}$. On the other hand, Kovtun et al. [7] conjectured $\frac{\eta}{s} \geq \frac{\hbar}{4 \pi k_{B}}$ to be the lower bound (KSS bound) for a wide class of systems. Interestingly such a finite but low value of $\eta / s$ is found to be consistent with the analysis of RHIC data through hydrodynamical simulations [8, 9].

Thus heavy ion experiments suggest formation of QGP with a behavior of near perfect fluidity i.e. very low viscosity. There are different $(2+1) \mathrm{d}$ [10 [13] and $(3+1) \mathrm{d}$ [14, 15] viscous hydrodynamic codes which are used to estimate QGP viscosity from the experimental data using the elliptic flow coefficient, $v_{2}$. The initial spatial deformation of the fireball created in relativistic heavy-ion experiments is converted into final state momentum anisotropies through hydrodynamic simulations. Viscosity comes into play via the degradation of this conversion efficiency. As in experimental detections only the final state hadrons are tracked, the most efficient observable to be related to these studies is the charged hadron elliptic flow, $v_{2}^{c h}$. Therefore the best description is provided with the amalgamation of the viscous hydrodynamic approach to the QGP phase and a microscopic description for re-scattering of late hadronic stage. Such hybrid approaches include VISHNU [16] which incorporates VISH2+1 [10, 17] algorithm with UrQMD cascade model [18] and McGill code which connects $(3+1) \mathrm{d}$ viscous hydrodynamics to UrQMD. The pioneering study in this regard was carried out by Luzum and Romatschke [12] using $(2+1) d$ viscous hydrodynamics. One of the points of uncertainty in these studies is initial condition. Different initial conditions like in MC-KLN or MC-Glauber lead to uncertainties in the values of $\left(\frac{\eta}{s}\right)_{Q G P}$ by factor of 2 to 2.5 [19]. A recent study carried out using MUSIC + UrQMD [20], with the IP-Glasma initial conditions [21], shows excellent match to multiplicity and flow distributions at RHIC and LHC. In fluid dynamical descriptions of the created fireball, shear viscosity to entropy density ratio $\frac{\eta}{s}$ is usually taken to be temperature independent. Predictions made in order to explain the azimuthal anisotropies of the spectra, like the elliptic flow coefficient $v_{2}$ reveal very small value for this $\frac{\eta}{s} \sim 0.1[11,12$, 22, 23]. However, there are some works including the temperature dependence of $\frac{\eta}{s}$ 24] as well, where the authors have taken different parameterizations to get a thorough understanding of the effect on elliptic flow as well as higher harmonics. Lattice studies of transport coefficients of a gluon plasma have been carried out using $16^{3} \times 8$ and $24^{3} \times 8$ lattices 25] indicating an ideal fluid behavior of QGP.

There are different techniques which can be used for the evaluation of $\eta$ in strongly interacting systems, namely, the Relaxation Time Approach [26], the Chapman-Enskog method [27] and the Green-Kubo formalism [28]. In Relaxation Time Approach (RTA), it is assumed that the collisional effects drive the perturbed distribution function close to the equilibrium one with a relaxation time of the order of the time required for particle collisions. On the other hand, the Chapman-Enskog approximation is based on the fact that on slight shift of the distribution function from its equilibrium value, the former can be expressed in terms of hydrodynamical variables and their gradients. One advantage of Chapman-Enskog method is that one can do a successive approximation to get results closer to Kubo formalism. The Green-Kubo formalism relates linear transport coefficients to near-equilibrium correlations of dissipative fluxes and treats them as perturbations to local thermal equilibrium. A comparative study of the three different methods has been carried out by Wiranata et al. [29]. In the varied cases considered in this paper, the GreenKubo technique is found to be more reliable. In [30] it has been argued that while in the case of CE, variational method may yield solutions with arbitrary accuracy depending on the order of approximation, RTA has no control over its accuracy. The RTA result was found to differ from that obtained using Green-Kubo formalism by factor of 2. On the other hand, the CE method, already at 1st order, was found to display satisfactory agreement with the Green-Kubo results. Comparisons in the context of the non-relativistic hard sphere can be found in classical literature [31], from which it is inferred that higher order approximations of the CE method approaches the Green-Kubo one. Notwithstanding this fact the RTA method has often been used due to its simplicity. It has been used to evaluate $\eta$ for two flavor matter in NJL model [32]. On the other hand, in [33], a combination of large $N_{c}$ expansion and Kubo formalism was used to calculate $\eta$ in two flavor NJL model. In the present work we have used the framework of 2+1-flavored Polyakov-Nambu-Jona-Lasinio (PNJL) model to study the behavior of $\eta$ at finite temperature and density. The variation of $\eta$ with temperature and density is then used to discuss the location of critical end point (CEP).

The present article is organized as follows. The outline of the formalism adopted for this work is given in Section II followed by a brief introduction to the PNJL model in Section III. In Section IV-A, we look for the variational nature of $\eta$ with $T$ for different choices of the spectral width $\Gamma$. Further, we compute $\eta$ as a function of quark chemical potential $\mu_{q}$ for three different choices of $T$, viz. one in the expected range of 1st order phase transition, other in cross-over range and finally the last one beyond the cross-over region. We also discuss the variation of $\frac{\eta}{s}$ as a function 
of $\mu_{q}$ for a wide range of $T$. In Section IV-B, variation of $\frac{\eta}{s}$ with $T$ at various $\mu_{q}$ has been used to draw the phase diagram and identify the CEP region. The location of CEP is further validated with the behavior of the specific heat, $C_{V}$ in Section IV-C . In Section IV-D, we calculate $\frac{\eta}{s}$ under different experimental conditions considering the freeze-out parameterization. Finally, the results are summarized in Section V.

\section{KUBO FORMALISM}

Kubo formalism, as mentioned earlier, calls for the spectral width of the degrees of freedom of the system involved. This is realized through the fact that, shear viscosity coefficient $\eta$ is related to retarded correlators of energy-momentum (E-M) tensor i.e. 4-point functions in Matsubara space. The energy-momentum function is defined as :: $T_{\mu \nu}=$ $i \bar{\psi} \gamma_{\mu} \partial_{\nu} \psi-g_{\mu \nu} \mathcal{L}$. Making use of this energy-momentum tensor, Kubo formula for shear viscosity reduces to the form [33],

$$
\eta(\omega)=\frac{1}{15 T} \int_{0}^{\infty} d t e^{i \omega t} \int d \vec{r}\left(T_{\mu \nu}(\vec{r}, t), T^{\mu \nu}(0,0)\right),
$$

where $T_{\mu \nu}$ is the $(\mu, \nu)$ component of the E-M tensor of quark matter. The factor 15 in the above equation comes from the following identity [33],

$$
\int d^{3} x x_{i}^{2} x_{j}^{2} f\left(x^{2}\right)=\frac{1}{15} \int d^{3} x x^{4} f\left(x^{2}\right) .
$$

Starting from Eq.(1) and neglecting the surface terms at infinity, we arrive at the following expression of $\eta$ in terms of the retarded correlators:

$$
\eta(\omega)=\frac{i}{\omega}\left[\Pi^{R}(\omega)-\Pi^{R}(0)\right]
$$

Following the steps of [33, 34] we get,

$$
\eta=\frac{\pi}{15 T} \int_{-\infty}^{\infty} d \varepsilon \int \frac{d^{3} p}{(2 \pi)^{3}} p_{x}^{2} f_{\Phi}\left(1-f_{\Phi}\right) \operatorname{Tr}\left[\gamma_{2} \rho(\epsilon, p) \gamma_{2} \rho(\epsilon, p)\right]
$$

where, $f_{\Phi}$ is the distribution function of the fermionic fields in presence of the Polyakov loop $\Phi$ and $\rho(\omega, p)$ is the spectral function defined as,

$$
\rho(\omega, p)=\frac{1}{2 \pi i}\left(G^{A}(\omega, p)-G^{R}(\omega, p)\right)
$$

with $G^{R / A}$ being the advanced and retarded Green's function represented as,

$$
G^{R / A}=\frac{1}{\not p-M \pm i \operatorname{sgn}\left(p_{0}\right) \Gamma(p)} .
$$

In the conventional notation, $M$ is the quasi-particle mass and $\Gamma(p)$, the spectral width. Incorporating simple algebraic techniques and using Eq.(4) and Eq.(5), we finally arrive at,

$$
\eta[\Gamma(p)]=\frac{16 N_{c} N_{f}}{15 \pi^{3} T} \int_{-\infty}^{\infty} d \varepsilon \int_{0}^{\infty} d p p^{6} \frac{M^{2} \Gamma^{2}(p) f_{\Phi}(\varepsilon)\left(1-f_{\Phi}(\varepsilon)\right)}{\left(\left(\varepsilon^{2}-p^{2}-M^{2}+\Gamma^{2}(p)\right)^{2}+4 M^{2} \Gamma^{2}(p)\right)^{2}}
$$

where, $N_{c}$ and $N_{f}$ are the no. of colors and flavors respectively.

\section{PNJL MODEL}

The Polyakov-Nambu-Jona-Lasinio (PNJL) Model 35 39] is a QCD-inspired phenomenological model developed by coupling the Polyakov loop potential to the Nambu-Jona-Lasinio (NJL) model. In the NJL model, the interactions between quarks is accounted for by four quark terms which respect the chiral symmetry of the Lagrangian. Spontaneous breaking of chiral symmetry takes place due to dynamical generation of fermion mass. Since the gluons are integrated out, the NJL model fails to simulate the deconfinement physics. Because of its failure to incorporate 
confinement, some important aspects of the QCD thermal transition are not suitably accounted for in the NJL model. However unlike NJL model, the PNJL encapsulates this feature of the QCD transition. In this model, the gluon dynamics is described by the background temporal field. So, here the chiral and deconfinement order parameters are entwined into a single framework. Several quantities like pressure, number density, speed of sound etc. calculated in this model show very good agreement with lattice results [39 41]. In the present work we have considered $S U(3)_{f}$ version of PNJL model including 8-quark interaction [42]. The current quark masses used here are $m_{u}=m_{d}=8.7$ $\mathrm{MeV}$ and $m_{s}=179.5 \mathrm{MeV}$ along with the three momentum cutoff $\Lambda=640 \mathrm{MeV}$ [42].

While computing the shear viscosity $\eta$ as in Eq.(7), we incorporated the modified Fermi-Dirac distribution functions $\left(f_{\Phi}\right)$ in which the effect of the background Polyakov loop fields is taken into account. The forms of the distribution functions for the particles and anti-particles as realized in PNJL model are ::

$$
\begin{aligned}
& f_{\Phi}^{+}\left(E_{p}\right)=\frac{\left(\bar{\Phi}+2 \Phi e^{-\beta\left(E_{p}+\mu\right)}\right) e^{-\beta\left(E_{p}+\mu\right)}+e^{-3 \beta\left(E_{p}+\mu\right)}}{1+3\left(\bar{\Phi}+\Phi e^{-\beta\left(E_{p}+\mu\right)}\right) e^{-\beta\left(E_{p}+\mu\right)}+e^{-3 \beta\left(E_{p}+\mu\right)}}, \\
& f_{\Phi}^{-}\left(E_{p}\right)=\frac{\left(\Phi+2 \bar{\Phi} e^{-\beta\left(E_{p}-\mu\right)}\right) e^{-\beta\left(E_{p}-\mu\right)}+e^{-3 \beta\left(E_{p}-\mu\right)}}{1+3\left(\Phi+\bar{\Phi} e^{-\beta\left(E_{p}-\mu\right)}\right) e^{-\beta\left(E_{p}-\mu\right)}+e^{-3 \beta\left(E_{p}-\mu\right)}},
\end{aligned}
$$

where, ' \pm ' refer to the particle and anti-particle respectively. $\Phi \& \bar{\Phi}$ are the Polyakov loop fields, $\mu$ and $\beta$ being the chemical potential and inverse temperature respectively. Starting from PNJL thermodynamic potential we can calculate the fields (degrees of freedom of the system involved), pressure and constituent masses at corresponding temperatures and chemical potentials. The details of the technique for 2 and $2+1$ flavor cases can be found in [38, 39] and [42, 43] respectively. With these inputs, we proceed to determine $\eta$ and $s$.

\section{RESULTS}

\section{A. Parameterization of $\Gamma$}

The spectral width $\Gamma(\mathrm{p})$, in PNJL model, is supposed to have contributions from Landau damping of quarks and mesons along with the recombination processes i.e. the formation of collective mesonic modes due to quark - antiquark rescattering. Spectral width for 2 flavor case including both $\sigma$ and $\pi$ has been evaluated at next-to-leading order in the large $N_{c}$ expansion including one-loop mesonic contributions [33, 44]. Spectral widths evaluated this way would depend on both $T$ and $\mu$. In the case of $S U(3)_{f}$ PNJL model all the scalar and pseudoscalar meson channels will contribute to this process. Moreover, the decay widths themselves may become comparable or larger than mass, especially at lower temperatures. Under such circumstances, one should really express $\eta$ explicitly in terms of spectral functions which should then be evaluated considering all possible channels [45]. Since such a calculation is extremely involved, in our present work, we have considered the forms of $\Gamma(p)$ as given in [33]. The configurations of $\Gamma$ proposed to ensure the convergence of $\eta$ are::

$$
\begin{gathered}
\text { Constant }: \Gamma_{\text {const }}=100 \mathrm{MeV}, \\
\text { Exponential }: \Gamma_{\exp }=\Gamma_{\text {const }} e^{-\beta p / 8}, \\
\text { Lorentzian }: \Gamma_{\text {Lor }}(p)=\Gamma_{\text {const }} \frac{\beta p}{1+(\beta p)^{2}}, \\
\text { Divergent }: \Gamma_{\text {div }}(p)=\Gamma_{\text {const }} \sqrt{\beta p} .
\end{gathered}
$$

Fig.(11) shows the variation of $\eta$ with $T$, at $\mu_{q}=0$, for different choices of $\Gamma$ as given earlier, whereas in the inset result considering 2 flavors for a constant $\Gamma=100 \mathrm{MeV}$ is shown. For a given $\Gamma, \eta$ is found to increase with temperature. This behavior is similar to a gaseous system where viscosity increases with temperature due to the increase in the average momentum of the particles [46]. In the present case, the increase in $\eta$ may be attributed to the decrease in the quark mass with temperature in our model. Fig.(1) shows that $\eta$ becomes very small in the low temperature range. In this low temperature region, the quark masses become large and $\eta$ is expected to fall as $M^{-6}$ [33]. It can also be seen that $\eta_{\text {Lor }}>\eta_{\text {exp }}>\eta_{\text {const }}>\eta_{\text {div }}$. This behavior simply depends on the value of $\Gamma$ at a given temperature. A lower value of $\Gamma$ corresponds to weaker interaction and hence a larger mean free path [33]. It is evident fom Eq. (77) that the $\eta$ for 2 -flavor matter will be less than the 3-flavor for equal masses. Since, $s$ quark mass is higher than the $u$ and $d$ masses, the difference is less than the equal mass case. In the inset of Fig.(11) we have shown $\eta$ for 2 -flavor matter evaluated in PNJL with the parameter set adopted in [40, 41].

Variation of $\eta$ with $\mu_{q}$ is shown in Fig.(2). Here we have chosen three different temperatures $\mathrm{T}=100,150$ and 200 $\mathrm{MeV}$, corresponding to the 1st order phase transition, cross-over and beyond cross-over regions. Similar to the zero chemical potential case, Fig.(2) also shows increase in $\eta$ with $\mu_{q}$ at fixed $T$. However, the nature of the curves are 


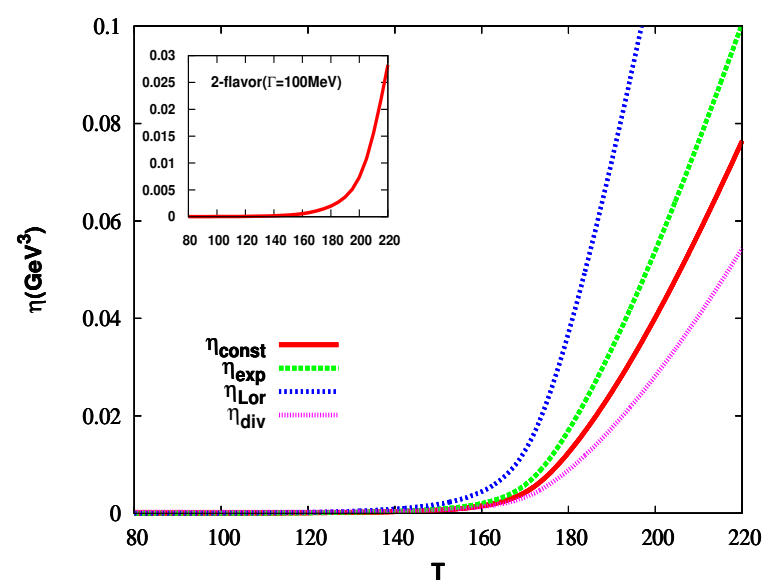

FIG. 1: (color online) $\eta$ as a function of temperature at vanishing chemical potential for different forms of $\Gamma$

different for the three different choices of temperature. For $\mathrm{T}=100 \mathrm{MeV}$, moving along the $\mu$-axis, one is expected to encounter the 1st order phase transition line. Here, $\eta$ shows a jump for all forms of $\Gamma$ at $\mu_{q} \simeq 280 \mathrm{MeV}$. On the other hand, both for $\mathrm{T}=150 \mathrm{MeV}$ and $\mathrm{T}=200 \mathrm{MeV}$ Fig.(2) shows, as expected, a smooth variation in $\eta$ along $\mu$-direction. Moreover, $\eta$ at finite $\mu_{q}$ is larger than that for $\mu_{q}=0$ and increases substantially for larger $T$.
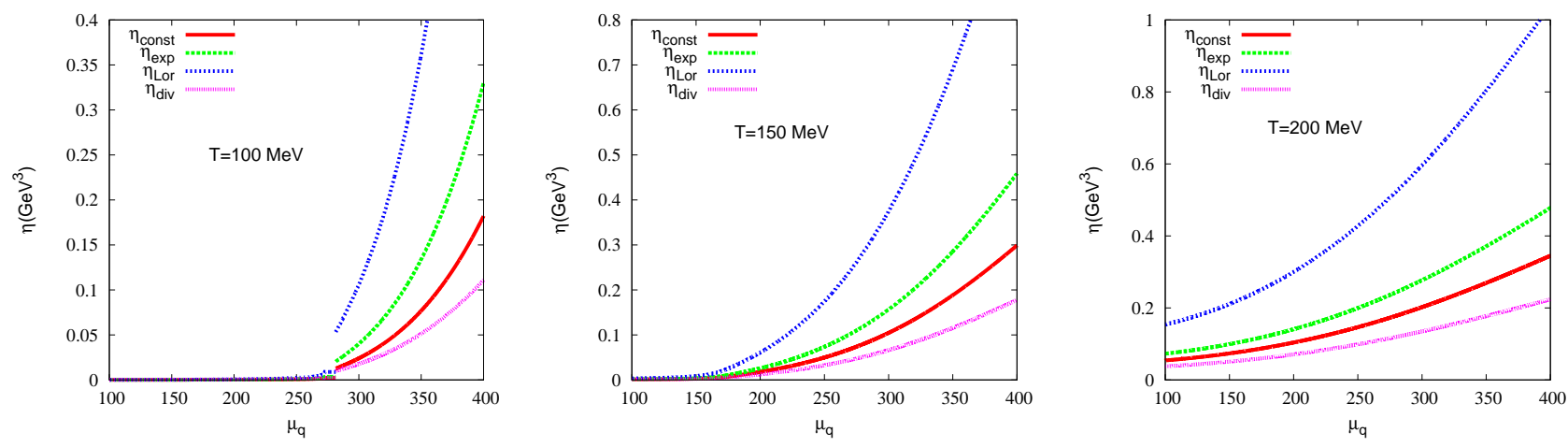

FIG. 2: (color online) $\eta$ as a function of quark chemical potential at fixed temperatures for different forms of $\Gamma$

It has already been mentioned that smaller $\Gamma$ correspond to a larger $\eta$. Hence the different forms of $\Gamma$, as observed earlier, only affect the rate at which $\eta$ changes with $T$ and $\mu_{q}$. But these forms do not change the qualitative behavior of $\eta$, as seen from Figs.(11)-(2). As mentioned earlier, in an explicit calculation one should evaluate the spectral width $\Gamma(\mathrm{p})$ considering the contributions from all meson channels in $S U(3)_{f}$ PNJL model. This $\Gamma(\mathrm{p})$ will depend on both $T$ as well as $\mu$. In the absence of such a rigorous evaluation, we would use the sum rule essential for the choice of Breit-Wigner form, as a guiding principle [34, 47],

$$
\frac{1}{4} \operatorname{Tr}_{\text {spin }} \int_{-\infty}^{\infty} \frac{d \epsilon}{2 \pi}\left[\rho(\epsilon, p) \gamma_{0}\right]=1
$$

The Eq.(10) is satisfied approximately when the value of $\Gamma \leq M$. So for our further discussions we choose a constant spectral width $\Gamma_{\text {const }}=100 \mathrm{MeV}$ so that the sum rule is in general violated to a considerable extent only in the low momentum region. In fact, as the quark masses drop sharply around $T_{c}$, from its $T=\mu_{q}=0$ value, the violation is expected to be more pronounced for $T>T_{c}$ only. But again the smaller contribution from low momentum at these high temperatures keeps the violation to a minimum level. For $\Gamma>100 \mathrm{MeV}, \eta$ variation is similar to that for $\Gamma_{d i v}$, whereas, for $\Gamma<100 \mathrm{MeV}$, it is similar to that for $\Gamma_{\text {Lor }}$. At the same time, in the regime of momentum transfer comparable to QCD-scale $(\sim 200 \mathrm{MeV}), \Gamma(p)$ becomes $\sim 100 \mathrm{MeV}$ considering contributions from one-loop mesonic channels at next-to-leading order in the large $N_{c}$ expansion as shown in [33]. There, the authors have included Landau 
damping and recombination process as the leading dissipative effects to calculate shear viscosity at the one loop level taking all mesons influencing the spectral width. However going further up in the quark-momentum leads towards decrease in spectral width which in turn increases the shear viscosity.

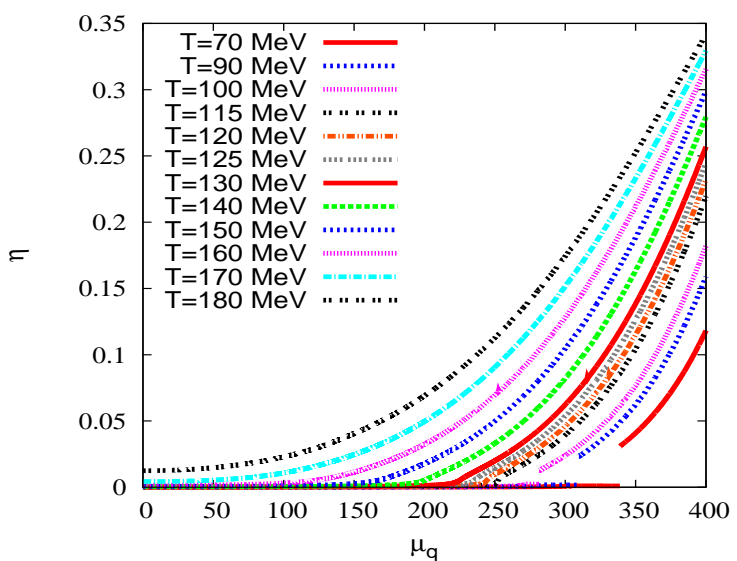

FIG. 3: (color online)Variation of shear viscosity with quark chemical potential at different temperatures

Let us again look at the variation of $\eta$ with $\mu_{q}$ for different values of $T$ with $\Gamma=100 \mathrm{MeV}$. From Fig.(3), two distinct regimes are clearly visible. For $\mathrm{T}=70$ to $100 \mathrm{MeV}$, we get a jump in $\eta$, whereas for $\mathrm{T}=120$ to $180 \mathrm{MeV}, \eta$ has a smooth behavior analogous to the results displayed in Fig.(2). The temperature range $\mathrm{T}=70-100 \mathrm{MeV}$ and 120 $180 \mathrm{MeV}$ lie in the first order and cross-over zones of transition respectively and Fig.(3) shows anticipated outcome of $\eta$ with $\mu_{q}$. The region around $\mathrm{T} \simeq 115 \mathrm{MeV}$ calls for special attention and will be discussed in details in the upcoming section.

\section{B. $\eta / \mathrm{s} \&$ the Phase Diagram}

In general $\eta$ for different fluids vary widely differing by orders of magnitudes [46]. In such circumstances, Reynolds number, the ratio of inertial to viscous forces in the Navier-Stokes equation, is traditionally used as a measure of fluidity. In the case of relativistic fluids, the Reynolds number (more specifically its inverse) may be defined in terms of $\eta / \mathrm{s}, \mathrm{s}$ being the entropy density. Here we present this ratio in Fig.(4) for $\mu_{q}=0$ for $2+1$ flavor quark matter in PNJL model. We have shown comparative plots for different constant values of the spectral width to get a better understanding of the effect of $\Gamma$ on the specific shear viscosity, $\frac{\eta}{s}$. The results are compatible with the arguments presented in the last section. $\frac{\eta}{s}$ has been evaluated in NJL model in [33] using Kubo formalism whereas in [32] the ratio was obtained using relaxation time approach. Our results in the PNJL model are qualitatively similar to those obtained in 33].

As shown in Fig. (4) $\frac{\eta}{s}$ starts from high values at low temperatures and decreases to a minimum of $\frac{1}{4 \pi}$ corresponding to ideal fluids near $T=T_{c}$. As discussed earlier, $\eta$ itself is extremely small for $T<T_{c}$. The behavior of $\frac{\eta}{s}$ for this region of temperature is due to a larger drop in entropy density of the system. A simple calculation for pion gas shows $\frac{\eta}{s}$ to be proportional to $\left(\frac{f_{\pi}}{T}\right)^{4}$ where, $f_{\pi}$ is the pion decay constant [48, 49]. Hence, for $\mathrm{T} \rightarrow 0, \frac{\eta}{s}$ should diverge. Similar results have also been obtained by Lang et. al. [50] who have computed $\frac{\eta}{s}$ for interacting pion-gas considering different pion masses. In the inset of Fig.(4), the region from $\mathrm{T}=80$ to $140 \mathrm{MeV}$ has been zoomed in for a better comparison with the system of interacting pion gas [50]. A comparison of our results in this temperature region with corresponding result for meson gas $\left(T<T_{c}\right)$ obtained from chiral perturbation theory [51] is also shown in Fig. (4).

In general, for an ideal gas of quarks, the $\eta$ and hence $\frac{\eta}{s}$ should diverge at large $T$ limit. As shown in Fig.(4) $\frac{\eta}{s}$ does show an increasing trend for $T>T_{c}$. The behavior of $\frac{\eta}{s}$ up to about $T \sim 1.5 T_{c}$ seems to closely resemble the features of a fluid having liquid-gas phase transition, for which a minima is expected near the transition point [46, 54]. However, since all the quark masses drop to their respective current masses for $T>1.5 T_{c}$, entropy starts dominating. As a result $\frac{\eta}{s}$ starts decreasing slowly with increasing $T$, and hence displays a behavior of an interacting liquid. In lattice studies similar behavior has been observed for pure glue plasma (GP) as shown in Fig.(44). The results of perturbation theory [52, 53] is around 1 as both $\eta$ and $s$ varies as $T^{3}$ at higher temperatures. Eventually for asymptotic temperatures an ideal gas behavior is expected to be restored. 


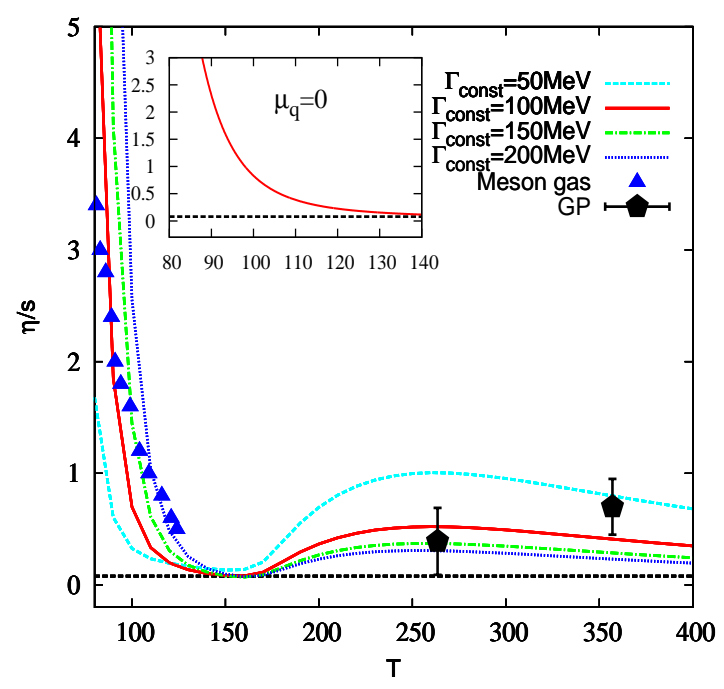

FIG. 4: (color online)Specific shear viscosity $\left(\frac{\eta}{s}\right)$ as a function of temperature at vanishing chemical potential

Similar features are observed with the increase in $\mu_{q}$ as shown in Fig.(5). To summarize the situation we see that for $\mu_{q}=0, \frac{\eta}{s}$ initially decreases with increase in $T$, reaches the minimum of $\frac{1}{4 \pi}$ bound near $T_{c}$. Thereafter it increases with $T$ rather slowly after transition before stabilizing/ slowly decreasing in accordance with the behavior found in Ref.[25, [55, [56]. As we increase $\mu_{q}$, the same feature is observed for $\mu_{q} \leq 100-150 \mathrm{MeV}$. On the other hand, for $\mu_{q} \geq$ $200 \mathrm{MeV}$, the system undergoes a transition at lower temperatures and $\frac{\eta}{s}$ has a minimum which is significantly higher than the KSS bound. Moreover, for $\mu_{q} \geq 260 \mathrm{MeV}, \frac{\eta}{s}$ shows a jump which may be attributed to a first order phase transition. Therefore a possibility of observing a CEP arises near these ranges of $T$ and $\mu_{q}$.
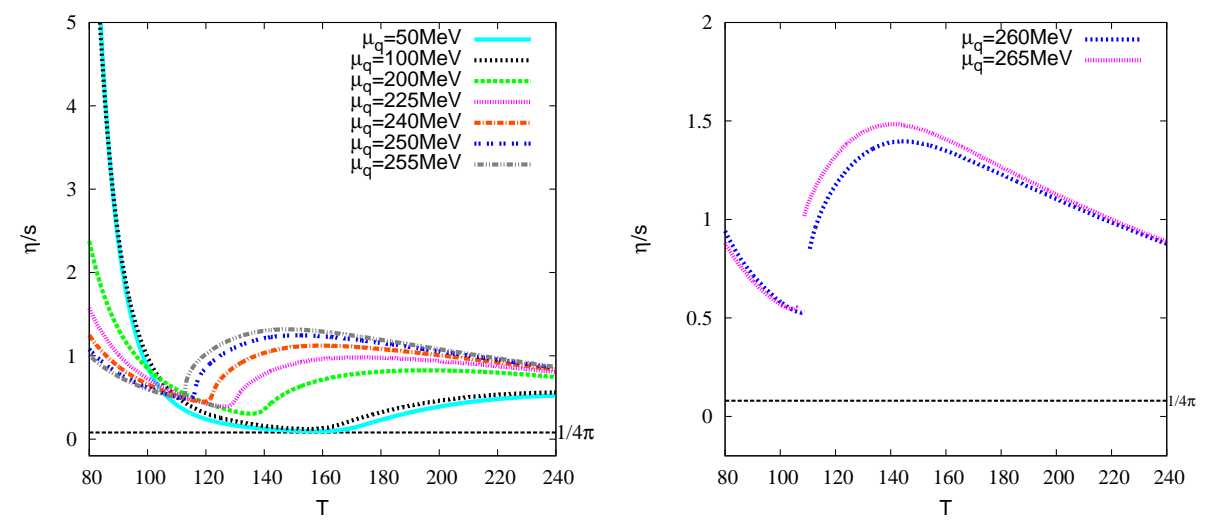

FIG. 5: (color online) $\frac{\eta}{s}$ as a function of temperature for different non-zero values of quark chemical potential

QCD critical end point is supposed to be described by model $\mathrm{H}$ [57]. $\eta$ is expected to diverge, with a small power of correlation length, near the CEP [58, 59]. On the other hand, for weakly coupled real scalar field theories, $\frac{\eta}{s}$ is most likely to develop a cusp at CEP [60]. A discontinuity in the behavioral pattern of $\frac{\eta}{s}$ around CEP region has also been discussed in 61.

In our study the location of the minima and the discontinuities of $\frac{\eta}{s}$ in Fig.(15), enable us to extract the critical values of $T \& \mu$ to draw the Phase-diagram. However in the very low temperature region extrapolation has been done considering the fitting function in the form of polynomial

$$
T=a_{0}+a_{1} \mu+a_{2} \mu^{2}
$$

with, $a_{0}=50 \mathrm{MeV}, a_{1}=-2.5$ and $a_{2}=-0.04 \mathrm{MeV}^{-1}$. The phase diagram along with the CEP region (black dot) has been plotted in Fig.(15). 


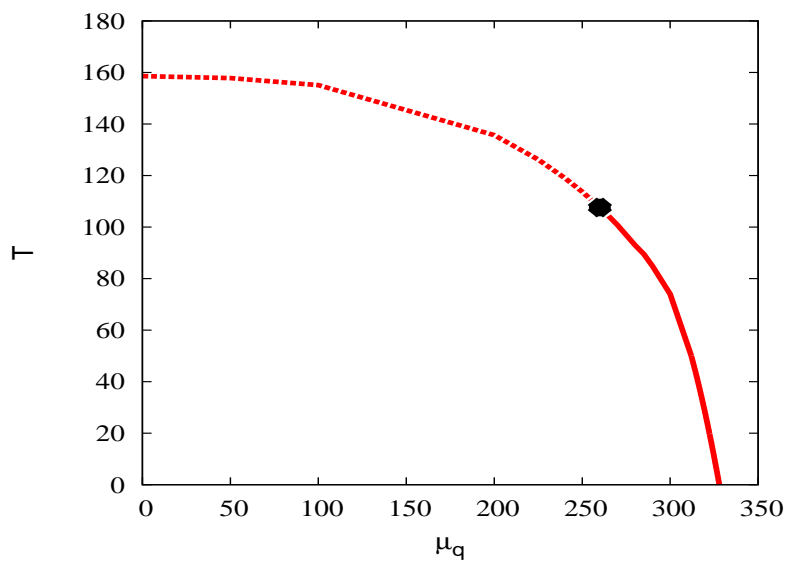

FIG. 6: (color online)Phase diagram for 2+1 flavor PNJL Model

The understanding of the behavior of the strongly interacting system near CEP along with its location is extremely important. Various efforts are being undertaken, both theoretically as well as experimentally, to determine the position of CEP [55, 62]. In general, second order derivatives of thermodynamic quantities are expected to diverge near CEP which is a second order transition point. These quantities may provide additional information regarding the CEP. Here, in the PNJL model, we have observed that at or around CEP, wide variations in order parameters, fluctuations of conserved charges like net electric charge [63] can occur as far as dynamic as well as static properties of the system are concerned.

\section{On the location of CEP}

In order to verify our results, we have considered the specific heat $C_{V}$, which is expected to show diverging behavior near the CEP. One can define $C_{V}$ as ::

$$
C_{V}=\frac{\partial \epsilon}{\partial T}=T \frac{\partial^{2} P}{\partial T^{2}}=T \frac{\partial s}{\partial T}
$$

The divergence in $C_{V}$ near the CEP will translate into highly enhanced transverse momentum fluctuations or highly suppressed temperature fluctuations for a system passing close to the CEP. In Fig.(17), we have shown the variation
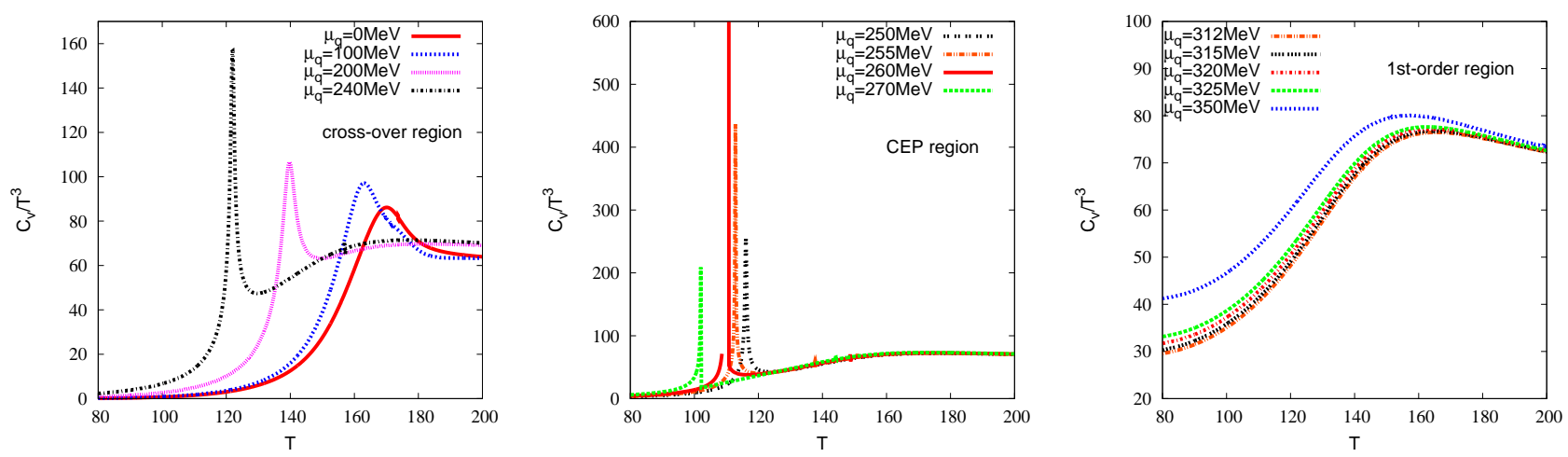

FIG. 7: (color online) $C_{V} / T^{3}$ as a function of temperature for different values of $\mu_{q}$

of dimensionless quantity $\frac{C_{V}}{T^{3}}$ with $\mathrm{T}$ separately in the regions of cross-over, CEP, 1st order and beyond, along the $\mu_{q}$ direction. As is seen $\frac{C_{V}}{T^{3}}$ shoots up around $\mu_{q} \sim 260 \mathrm{MeV}$, but shows continuous behavior elsewhere. Thus we confirm that the CEP is expected to exist around $T=100-120 \mathrm{MeV}$ and $\mu_{q}=250-270 \mathrm{MeV}$ as inferred from the behavior of $\frac{\eta}{s}$ in the previous subsection. 


\section{Connection with experiments}

In experiments, the observables are studied as a function of center of mass collision energy $(\sqrt{s})$. In our thermodynamic studies the independent variables are $T, \mu_{B}, \mu_{Q}$ and $\mu_{S}$ where $\mu_{B}, \mu_{Q}$ and $\mu_{S}$ are the the baryon, electric charge and strangeness chemical potentials respectively. So to get the collision energy dependence one needs to get a parameterization between $\sqrt{s}$ and various thermodynamic variables. Different parameterizations of the freeze-out conditions as function of $\sqrt{s}$ are available in the literature. For a given set of the thermodynamic variables, the variations in $\sqrt{s}$ are within $10 \%$ for different parameterizations. In the present study we have used the following parameterization [64]:

$$
T\left(\mu_{B}\right)=a-b \mu_{B}^{2}-c \mu_{B}^{4} \& \mu_{B, Q, S}(\sqrt{s})=\frac{d}{1+e \sqrt{s}}
$$

where, $a=(0.166 \pm 0.002) G e V, b=(0.139 \pm 0.016) G e V^{-1}, c=(0.053 \pm 0.021) G e V^{-3}$ and $d$ and $e$ are given by :

\begin{tabular}{|c|c|c|}
\hline & $d[\mathrm{GeV}]$ & $e\left[\mathrm{GeV}^{-1}\right]$ \\
\hline $\mathrm{B}$ & $1.308(28)$ & $0.273(8)$ \\
\hline $\mathrm{Q}$ & 0.0211 & 0.106 \\
\hline $\mathrm{S}$ & 0.214 & 0.161 \\
\hline \hline
\end{tabular}

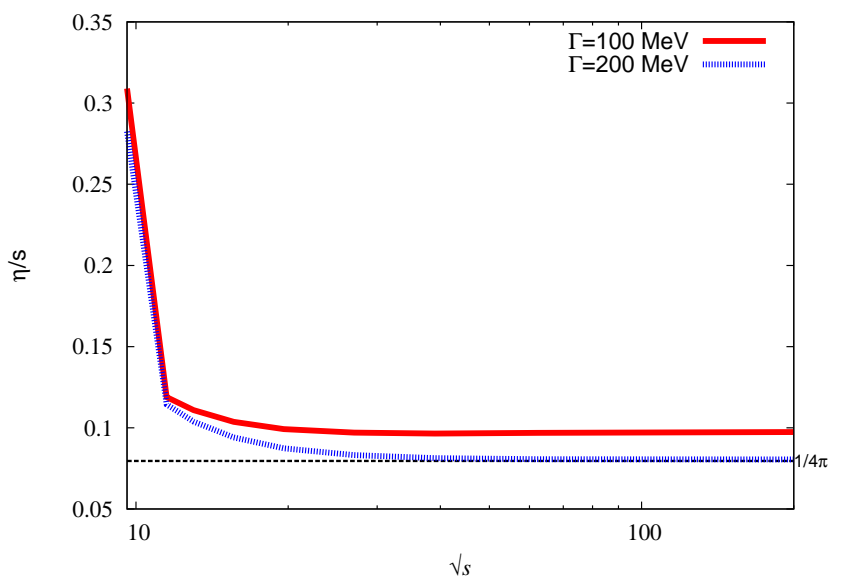

FIG. 8: (color online) $\frac{\eta}{s}$ under different experimental conditions along the freeze-out diagram

The variation of $\frac{\eta}{s}$ as a function of $\sqrt{s}$ along the freeze-out curve is displayed in Fig.(8). We have already seen that increase in $\Gamma$ results in the decrease in $\eta$. This is true for non vanishing chemical potential as well. On the other hand, at very high energies, $\frac{\eta}{s}$ is expected to maintain the specified lower limit or KSS bound of $\frac{1}{4 \pi}$. In Fig. (8), we have plotted specific shear viscosity considering a spectral width of 100 and $200 \mathrm{MeV}$. It can be seen that $\frac{\eta}{s}$ saturates to the KSS bound for $\Gamma=200 \mathrm{MeV}$ whereas for $\Gamma=100 \mathrm{MeV}$ saturation occurs at higher values. So, in the present study, the stronger interaction (in terms of $\Gamma$ ) seems to be necessary to attain the KSS bound.

It should be noted that the $\frac{\eta}{s}$ plotted here are for a system in complete thermodynamic equilibrium at a given value of temperature and chemical potential. Moreover, along the freeze-out curve, we always reside in the hadronic phase. So, the value of $\frac{\eta}{s}$ in Fig. (8) corresponds to different conditions in the hadronic sector. Initially $\frac{\eta}{s}$ decreases with $\sqrt{s}$ and then saturates at a value close to the KSS bound for higher $\sqrt{s}$. This is a reflection of the variation of $\frac{\eta}{s}$ as shown in Fig.(4) and Fig.(5). Our results as shown in Fig. (8) is close to $\frac{\eta}{s} \sim 0.09 \pm 0.015$ [55, 65] as extracted from the RHIC data for Au+Au collisions. Gavin and Abdel-Aziz [66] estimated, from the STAR data analysis, $\frac{\eta}{s}$ to lie in the range of 0.08-0.3. Another estimate done for top RHIC energies render a value of $\frac{\eta}{s}=0.12$ [67] which is also close to our model results. 
At RHIC, large azimuthal anisotropy of transverse momentum $\left(p_{T}\right)$ spectra, often expressed by the elliptic flow coefficient $v_{2}$ has been observed and this is considered to be a signature for the formation of QGP. The observed transverse momentum spectra of the hadrons and their centrality dependence [8, 21 24, 68] in relativistic heavy ion collision experiments can be explained satisfactorily using fluid dynamic descriptions. In fact, the notion of small $\frac{\eta}{s}$ for QGP arose from analysis of RHIC data through hydrodynamical simulations [9, 11, 19, 23, 68] which indicate non-viscous fluid properties for QGP. Though there have been predictions from various hydrodynamic calculations and simulations regarding temperature-independent $\eta$, uncertainties occur because of different initial conditions used. For example, use of data from $\mathrm{Au}+\mathrm{Au}$ collisions at RHIC leads to $\left(\frac{\eta}{s}\right)_{Q G P} \sim 0.08$ with MC-Glauber initializations, whereas that with MC-KLN results in 0.16 [19]. Moreover, analysis at $\mathrm{LHC}$ energies for $\mathrm{Pb}+\mathrm{Pb}$ collisions provide a bit higher result $\sim 0.2$ with various parameterizations incorporating different initial conditions [24, 67].

\section{CONCLUSION}

In the present study PNJL model has been used to calculate shear viscosity $\eta$ at finite temperature and chemical potential using Kubo formalism. Effect of spectral width $\Gamma$ has been discussed and finally constant $\Gamma$ has been used to present rest of our results. We have also presented our model results for $\frac{\eta}{s}$ for $T \neq 0, \mu_{q}=0$ and $T \neq 0, \mu_{q} \neq 0$ cases. Behavior of $\frac{\eta}{s}$ with $T$ and $\mu_{q}$ has been used to locate the CEP. This location of CEP has also been validated using the variation of specific heat $C_{V}$ with $T$ and $\mu_{q}$. Finally we have studied the variation of $\frac{\eta}{s}$ with $\sqrt{s}$ to compare with the values extracted from the analysis of RHIC and LHC data existing in the literature.

The salient points of the present study are ::

- $\eta$ by itself seems to show a gas like behavior and increases with increasing temperature. It has a strong dependence on the spectral width $\Gamma$, especially at higher temperatures.

- At $\mu_{q}=0, \frac{\eta}{s}$, in the present model, seems to reproduce the pion gas feature at low temperature and QGP features at high temperatures. Near $T_{c}, \frac{\eta}{s}$ has a minima at the KSS bound. For $T>T_{c}, \frac{\eta}{s}$ initially increases and then starts decreasing with increasing temperature showing a liquid like behavior. Moreover it agrees with the results from interacting pion gas at low $T$ and lattice data for gluon plasma at high $T$.

- The effect of non zero $\mu_{q}$ is found to be interesting. At $T=100 \mathrm{MeV}$, up to $\mu_{q}=250 \mathrm{MeV} \eta$ remains almost same as that for $\mu_{q}=0$ and then increases sharply to about 0.155 at $\mu_{q}=400 \mathrm{MeV}$ which is more than order of magnitude higher than the $\mu_{q}=0$ case. For higher $T$ the value of $\mu_{q}$, for which $\eta$ retains the $\mu_{q}=0$ value, decreases. For example at $T=200 \mathrm{MeV}$ and $\mu_{q}=250 \mathrm{MeV}, \eta$ is twice that of its $\mu_{q}=0$ value.

- For non-zero $\mu_{q}$, the minimum value of $\frac{\eta}{s}$ is found to be higher for larger $\mu_{q}$ and does not reach the KSS bound for $\mu_{q} \geq 200 \mathrm{MeV}$.

- Variation of $\eta$ across the phase boundary depends strongly on the nature of phase transition. In the cross over region, $\frac{\eta}{s}$ changes continuously with $\mu_{q}$ whereas it shows a jump in the first order transition region. The change in the nature of variation, in going from cross over to first order, may be used to extract the information of CEP. According to present analysis, CEP seems to lie in the range $T=100-120 \mathrm{MeV}$ and $\mu_{q}=250-270 \mathrm{MeV}$.

- $\frac{\eta}{s}$ on the freeze out curve seem to agree with the values extracted from RHIC and LHC flow analysis. At lower $\sqrt[s]{s}$ hadronic features seem to dominate which may be verified at FAIR experiments.

It will be interesting to use the present $T$ and $\mu_{q}$ dependence of $\frac{\eta}{s}$ for flow analysis to reproduce the data and will be pursued in our future studies.

\section{Acknowledgments}

The authors would like to thank CSIR and DST for funding this work. S.U. thanks Avik Banerjee for few useful discussions regarding the framework of Kubo formalism. S.U. and K.S. acknowledge Sabyasachi Ghosh and Sarbani Majumder for useful suggestions.

[1] T. Matsui and H. Satz, Phys. Lett. B178, 416 (1986). 
[2] P Koch, B Mller and J Rafelski, Phys. Rep. 142, 167 (1986).

[3] H. Meyer-Ortmanns, Rev. Mod. Phys. 68, 473 (1996).

[4] H. Stoecker, Nucl.Phys. A 750 (2005) 121-147. P. Bozek and I. Wyskiel, Phys.Rev. C 81(2010) 054902. J. Steinheimer et. al. Phys. Rev. C 89, 054913 (2014).

[5] J. Y. Ollitrault, Phys. Rev. D46, 229 (1992).

[6] G. Policastro, Dan T. Son and A. O. Starinets, Phys. Rev. Lett. 87, 081601 (2001).

[7] P. Kovtun, D. T. Son and A. O. Starinets, Phys. Rev. Lett. 94, 111601 (2005).

[8] P. Huovinen and P. V. Ruuskanen, Annu. Rev. Nucl. Part. Sci. 56, 163 (2006).

[9] H. Niemi et. al., Phys. Rev. Lett. 106, 212302 (2011).

[10] H. Song and U. Heinz, Phys. Lett.B 658, 279 (2008); Phys. Rev. C 77, 064901 (2008).

[11] P. Romatschke and U. Romatschke, Phys. Rev. Lett. 99, 172301 (2007).

[12] M. Luzum and P. Romatschke, Phys. Rev. C 78, 034915 (2008).

[13] K. Dusling and D. Teaney, Phys. Rev. C 77, 034905 (2008); P. Bozek, Phys. Rev. C 81, 034909 (2010); A. K. Chaudhuri, J. Phys. G 37, 075011 (2010).

[14] B. Schenke, S. Jeon and C. Gale, Phys. Rev. Lett. 106, 042301 (2011).

[15] P. Bozek, Phys. Rev. C 85, 034901 (2012).

[16] H. Song, S.A. Bass and U. Heinz, Phys. Rev. C 83, 024912 (2011).

[17] C. Shen and U. Heinz, Phys. Rev. C 83, 044909 (2011).

[18] S. A. Bass et. al., Prog. Part. Nucl. Phys. 41, 255 (1998); M. Bleicher et. al., J. Phys. G 25, 1859 (1999).

[19] U. Heinz and R. Snellings, Annu. Rev. Nucl. Part. Sci. 63:123 (2013).

[20] S. Ryu, S. Jeon, C. Gale, B. Schenke and C. Young, Nucl. Phys. A 904-905, 389c-392c (2013).

[21] B. Schenke, P. Tribedy and R. Venugopalan, Phys. Rev. Lett. 108, 252301 (2012); Phys. Rev. C 86, 034908 (2012).

[22] H. Song, S.A. Bass, U. Heinz, T. Hirano and C. Shen, Phys. Rev. C 83, 054910 (2011).

[23] H. Song et. al., Phys. Rev. Lett. 106, 192301 (2011).

[24] H. Niemi, G.S. Denicol, P. Huovinen, E. Molnar and D.H.Rischke, Phys. Rev. C 86, 014909 (2012).

[25] A. Nakamura and S. Sakai, Phys. Rev. Lett. 94, 072305 (2005).

[26] P. Chakraborty and J. I. Kapusta, Phys. Rev. C 83, 014906 (2011).

[27] W. A. van Leeuwen, P. Polak, and S. R. de Groot, Physica 63, 65 (1973).

[28] R. Kubo and K. Tomita, J. Phys. Soc. Jpn. 9, 888 (1954); H. Nakano, Prog. Theor. Phys. 15, 77 (1956); R. Kubo, J. Phys. Soc. Jpn. 12, 570 (1957).

[29] A. Wiranata and M. Prakash, Phys. Rev. C 85, 054908 (2012).

[30] S. Plumari, A. Puglisi, F. Scardina and V. Greco, Phys. Rev. C 86, 054902 (2012).

[31] J. A. McLennan, Introduction to Non-equilibrium Statistical Mechanics, 1st ed. (Prentice-Hall, Englewood Cliffs, New Jersey, 1989). 
[32] S. Ghosh, A. Lahiri, S. Majumder, R. Ray and S. K. Ghosh, Phys. Rev. C88, 068201 (2013).

[33] R. Lang and W. Weise, Eur. Phys. J. A (2014) $50: 63$.

[34] M.Iwasaki, H. Ohnishi and T. Fukutome, arXiv : hep-ph/0606192v1 19 Jun 2006.

[35] P. N. Meisinger and M. C. Ogilvie, Phys. Lett. B 379163 (1996); Nucl. Phys. B (Proc. Suppl.) 47519 (1996).

[36] K. Fukushima, Phys. Lett. B 591277 (2004).

[37] E. Megias, E. R. Arriola, and L. L. Salcedo, Phys. Rev. D 74, 065005 (2006); 74, 114014 (2006); J. High Energy Phys. 01 (2006) 073.

[38] C. Ratti, M. A. Thaler and W. Weise, Phys. Rev. D 73, 014019 (2006).

[39] S. K. Ghosh, T. K. Mukherjee, M. G. Mustafa and R. Ray, Phys. Rev. D 77, 094024 (2008).

[40] S. K. Ghosh, T. K. Mukherjee, M. G. Mustafa and R. Ray, Phys. Rev. D, 73, 114007 (2006).

[41] S. Mukherjee, Munshi G. Mustafa and R. Ray, Phys. Rev. D, 75, 094015 (2007).

[42] A. Bhattacharyya, P. Deb, S. K. Ghosh and R. Ray, Phys. Rev. D 82, 014021 (2010).

[43] M. Ciminale, R. Gatto, N. D. Ippolito, G. Nardulli, and M. Ruggieri, Phys. Rev. D 77, 054023 (2008).

[44] D. Muller, M. Buballa and J. Wambach, Phys. Rev. D 81, 094022 (2010).

[45] P. Czerski, W. M. Alberico, S. Chiacchiera, A. De Pace, H. Hansen, A. Molinari and M. Nardi, arXiv: 0708.0174v2 [hep-ph].

[46] T. Schafer and D. Teaney, Rep. Prog. Phys. 72, 126001 (2009).

[47] W. M. Alberico, S. Chiacchiera, H. Hansen, A. Molinari and M. Nardi, Eur. Phys. J.A. 38, 97-103 (2008).

[48] J. I. Kapusta, "Relativistic Nuclear Collisions", Landolt-Bornstein New Series, Vol. I/23, ed. R. Stock (Springer-Verlag, Berlin Heidelberg 2010).

[49] M. Prakash, R. Venugopalan and G. Welke, Phys. Rep. 227, 321 (1993).

[50] R. Lang, N. Kaiser and W. Weise, Eur. Phys. J. A (2012) 48:109.

[51] J. W. Chen and E. Nakano, Phys. Lett. B 647 (2007) 371.

[52] P. Arnold, G.D. Moore and L. G. Yaffe, J. High Energy Phys. 0305 (2003) 051.

[53] J. P. Blaizot, E. Iancu and A. Rebhan, Phys. Rev. Lett. 83, 2906 (1999).

[54] J. Frenkel, Kinetic Theory of Liquids (New York : Dover) 1955, H. Eyring, J. Chem. Phys. 4 283 (1936), D. Tabor, Gases, Liquids and Solids 2nd edn. (Cambridge : Cambridge University Press) (1979).

[55] R. A. Lacey, N. N. Ajitanand, J. M. Alexander, P. Chung, W. G. Holzmann, M. Issah, A. Taranenko, P. Danielewicz and H. Staker, Phys. Rev. Lett. 98, 092301 (2007).

[56] H. B. Meyer, Phys. Rev. D 76, 101701 (2007).

[57] D. T. Son and M. A. Stephanov, Phys. Rev. D70, 056001 (2004).

[58] P. C. Hohenberg and B. I. Halperin, Rev. Mod. Phys. 49, 435 (1977).

[59] A. Onuki, Phys. Rev. E55, 403 (1997).

[60] J. W. Chen, M. Huang, Y. H. Li, E. Nakano and D. L. Yang, Phys. Lett. B670, 18 (2008).

[61] C. Sasaki and K. Redlich, Nucl. Phys. A 832, 62 - 75 (2010). 
[62] R. A. Lacey, N. N. Ajitanand, J. M. Alexander, P. Chung, J. Jia, A. Taranenko and P. Danielewicz, arXiv : 0708.3512v6 [nucl-ex] 9 May 2008.

[63] A. Bhattacharyya, S. Das, S. K. Ghosh, S. Raha, R. Ray, K. Saha and S. Upadhaya, arXiv:1212.6010 [hep-ph] 2012.

[64] F. Karsch and K. Redlich, Phys. Lett. B 695, 136 (2011).

[65] R. A. Lacey, Nucl. Phys. A 785: 122-127, (2007).

[66] D. Teaney, Phys. Rev. C 68, 034913 (2003); S. Gavin and M. Abdel-Aziz, Phys. Rev. Lett. 97, 162302 (2006).

[67] C. Gale, S. Jeon, B. Schenke, P. Tribedy and R. Venugopalan, Phys. Rev. Lett. 110, 012302 (2013).

[68] C. Shen et. al., Phys. Rev. C 82, 054904 (2010). 